\title{
Papel dos antidepressivos na fibromialgia
}

Sofia Almeida, ${ }^{*}$ Ângela Pimenta, ${ }^{* *}$ Diana Brigas, ${ }^{* * *}$ Isabel Costa, ${ }^{* * * *}$ Luísa Santos*****

\section{RESUMO}

Introdução: A fibromialgia caracteriza-se por dor crónica generalizada de etiologia desconhecida, fadiga, rigidez, perturbação do sono, depressão, ansiedade, cefaleias, enxaqueca, alteração do trânsito intestinal, dor abdominal e urgência miccional. Os critérios de diagnóstico, segundo o Colégio Americano de Reumatologia (1990), são dor generalizada associada a hipersensibilidade em pelo menos 11 de 18 pontos músculotendinosos. A sua prevalência é de $2 \%$ nos Estados Unidos.

Objectivo: Revisão da evidência científica recente sobre o papel dos antidepressivos na fibromialgia.

Fontes de Dados: MedLine/Pubmed e Sites de Medicina Baseada na Evidência.

Métodos de Revisão: Pesquisa de meta-análises (MA) e revisões sistemáticas (RS), publicadas entre 2000 e Julho de 2008, em português e inglês, utilizando as palavras-chave fibromyalgia, antidepressants, fibromyalgia treatment. Foi utilizada a taxonomia SORT para apresentação dos resultados.

Resultados: Dos 10 artigos encontrados, foram seleccionadas 3 MA e 4 RS. Verificou-se que o efeito dos antidepressivos tricíclicos é moderado, sendo maior no sono e menor na dor e rigidez. A ciclobenzaprina, um fármaco com uma estrutura tricíclica que actua como miorrelaxante, demonstrou melhorar a função global, sono e, de forma modesta, a dor. Os inibidores da recaptação da serotonina e noradrenalina, duloxetina e milnacipram, são eficazes na dor e qualidade de vida, a venlafaxina parece eficaz em doses altas. Dos inibidores da recaptação da serotonina, a fluoxetina é eficaz na dor, bem-estar e depressão, a paroxetina reduz o impacto da doença na qualidade de vida, sem efeito na dor, e o citalopram é ineficaz. Dos inibidores da monoamina oxidase, o pirlindol melhora a dor e o bem-estar, o moclobemide é ineficaz.

Conclusões: Os antidepressivos com benefício no tratamento da fibromialgia são amitriptilina, duloxetina, milnacipram (SORT A), fluoxetina, pirlindol (SORT B). A ciclobenzaprina, apesar de não actuar como antidepressivo, partilha da estrutura tricíclica da amitriptilina e demonstrou ser benéfico no tratamento da fibromialgia (SORT A). São necessários estudos de longa duração para determinar o benefício destes fármacos a longo prazo.

Palavras-chave: Fibromialgia; Antidepressivos; Tratamento da Fibromialgia.

\section{INTRODUÇÃO}

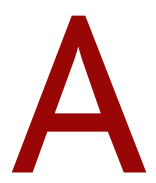

fibromialgia é uma síndrome comum, caracterizada por dor crónica generalizada de etiologia desconhecida. Alguns sintomas frequentemente associados incluem fadiga, rigidez, perturbação do sono, depressão, ansiedade, cefaleias, enxaqueca, alterações cognitivas, alteração do trânsito intestinal, dor abdominal difusa e urgência miccional. ${ }^{1}$

\footnotetext{
*Interna $3 .^{\circ}$ ano de MGF, C.S. Soares dos Reis e Oliveira do Douro - USF Nova Salus **Interna 3. ano de MGF, C.S. Castelo da Maia - USF Viver Mais

***Interna $3 .^{\circ}$ ano de MGF, C.S. Soares dos Reis e Oliveira do Douro - Unidade de Oliveira do Douro

****Interna $3 .^{\circ}$ ano de MGF, C.S. Soares dos Reis e Oliveira do Douro - USF Camélias *****Interna $3 .^{\circ}$ ano de MGF, USF Sete Caminhos - Gondomar
}

A prevalência estimada é de $2 \%$ na população dos Estados Unidos, sendo de 3,4\% nas mulheres e de $0,5 \%$ nos homens. ${ }^{2}$ Verifica-se um aumento da prevalência com a idade, atingindo $7 \%$ nas mulheres com idade igual ou superior a 70 anos. $^{3}$

Os critérios de diagnóstico de fibromialgia, definidos em 1990 pelo Colégio Americano de Reumatologia, são dor generalizada, definida como bilateral, atingindo as metades superior e inferior do corpo e incluindo a coluna vertebral e hipersensibilidade em pelo menos 11 de 18 pontos músculotendinosos específicos. ${ }^{2}$

Apesar de existirem diversas opções de tratamento para a fibromialgia, nenhuma é totalmente eficaz. Os antidepressivos têm sido muito usados no tratamento desta síndrome, por várias razões: serem eficazes nas perturbações do humor e da ansiedade, muito frequen- 
tes nestes doentes; coexistirem depressão major e fibromialgia em famílias, sugerindo a existência de mecanismos fisiopatológicos semelhantes nas duas patologias; haver evidência da eficácia dos antidepressivos que aumentam a neurotransmissão de noradrenalina e serotonina no tratamento da dor crónica; alguns antidepressivos mediarem o mecanismo anti-nociceptivo. ${ }^{4}$

Este estudo tem como objectivo rever a mais recente evidência científica sobre o papel dos antidepressivos na fibromialgia.

\section{MÉTODOS}

Foi efectuada uma pesquisa nas bases de dados Medline/Pubmed e sites de Medicina Baseada na Evidência, de meta-análises e revisões sistemáticas, publicadas entre Janeiro de 2000 e Julho de 2008, em português e inglês, com as palavras-chave: fibromyalgia, antidepressants, fibromyalgia treatment.

Para avaliar a qualidade dos estudos e a força de recomendação, foi utilizada a escala de Strength of Recommendation Taxonomy (SORT) da American Family Physician (AFP). Segundo esta taxonomia, a qualidade do estudo está subdividida em três Níveis de Evidência (Nível de Evidência 1: estudos de boa qualidade, evidência orientada para o doente; Nível de Evidência 2: estudos de qualidade limitada, evidência orientada para o doente; Nível de Evidência 3: outra evidência) e a força de recomendação em três graus (Força de Recomendação A: consistente, boa qualidade, evidência orientada para o doente; Força de Recomendação B: inconsistente ou qualidade limitada, evidência orientada para o doente; Força de Recomendação C: consensos, evidência orientada para a doença.

\section{RESULTADOS}

A pesquisa efectuada resultou na identificação de dez artigos, tendo sido excluídos três, por serem discordantes com o objectivo e incluídos sete, dos quais três meta-análises e quatro revisões sistemáticas.

Numa meta-análise de 2000, cujo objectivo era determinar a eficácia dos antidepressivos na fibromialgia e avaliar se esse efeito era independente da depressão, foram analisados treze ensaios clínicos aleatorizados controlados com placebo (EAC). ${ }^{5}$ Foram estudados antidepressivos tricíclicos, inibidores selectivos da recaptação da serotonina [ISRS] e um inibidor da monoami- noxidase [IMAO]. Nesta meta-análise, comprovou-se que os antidepressivos são eficazes em muitos sintomas da fibromialgia. O benefício foi moderado na dor, no sono e no bem-estar geral, embora pequeno na fadiga e no número de pontos dolorosos. Os doentes medicados com antidepressivo mostraram ter uma probabilidade de melhorar quatro vezes superior aos doentes medicados com placebo. Um em cada quatro doentes tratados melhorou a sintomatologia. Não foi possível, no entanto, determinar a eficácia relativa das diferentes classes de antidepressivos na fibromialgia, tal como não foi possível comprovar se a sua eficácia era independente do efeito antidepressivo (Nível de evidência 1). ${ }^{5}$

Numa outra meta-análise do mesmo ano, com o objectivo de determinar a eficácia dos antidepressivos tricíclicos na fibromialgia, foram analisados nove EAC. Nesta meta-análise, onde se encontra incluída a ciclobenzaprina, foi demonstrado que o efeito global dos antidepressivos tricíclicos na fibromialgia é moderado. O seu efeito foi maior na qualidade do sono e menor na sensibilidade dolorosa e na rigidez (Nível de evidência 1$){ }^{6}$

Em 2004, Tofferi JKet al realizaram uma meta-análise de cinco EAC com o objectivo de rever de forma sistemática a eficácia da ciclobenzaprina no tratamento da fibromialgia. A ciclobenzaprina é um fármaco com uma estrutura tricíclica semelhante à amitriptilina. No entanto, não tem um efeito antidepressivo, mas sim um efeito relaxante muscular. Este fármaco demonstrou oferecer algum benefício aos doentes com fibromialgia, melhorando a sua função global, a qualidade do sono e, de forma modesta, a dor. No entanto, não demonstrou melhorar a fadiga nem os pontos dolorosos. Ficou demonstrado que os doentes medicados com este fármaco têm uma probabilidade de melhorar três vezes superior aos doentes medicados com placebo. Um em cada cinco doentes tratados com ciclobenzaprina melhorou a sintomatologia (Nível de evidência 1). ${ }^{3}$

Em 2007, Lesley $M$ et alfez uma revisão sistemática sobre o uso de diversos antidepressivos no tratamento da fibromialgia. ${ }^{4}$ As principais conclusões dos diferentes estudos incluídos nessa revisão encontram-se descritas no Quadro I. De salientar que a duloxetina, um novo inibidor da recaptação da serotonina e da noradrenalina [IRSN], demonstrou melhorar significativamente a intensidade da dor, o número de pontos dolo- 


\begin{tabular}{|c|c|}
\hline Autor & Resultados \\
\hline $\begin{array}{l}\text { Arnold LM et al, } 2004 \\
\text { Arnold LM et al, } 2005\end{array}$ & $\begin{array}{l}\text { A duloxetina ( } 60 \mathrm{mg}, 2 \mathrm{id}) \text { melhorou o } \mathrm{n} .^{\circ} \text { de pontos dolorosos, a dor, a rigidez e a qualidade de vida. } \\
\text { O efeito na dor é independente do efeito no humor e nadepressão. (NE 1) }\end{array}$ \\
\hline Zijsltra TR et al, 2002 & A venlafaxina (75 mg 1id) melhorou a qualidade de vida e a fadiga, sem efeito na dor. (NE 1) \\
\hline $\begin{array}{l}\text { Dwight MM et al, } 1998 \\
\text { Sayar K, } 2003\end{array}$ & A venlafaxina demonstrou alguma eficácia em altas doses. (NE 2) \\
\hline Gendreau RM et al, 2005 & $\begin{array}{l}\text { O milnacipram ( } 25-200 \mathrm{mg} / \mathrm{dia}), 1 \text { id e } 2 \mathrm{id} \text {, melhorou a dor. O efeito foi superior nos que fizeram } \\
\text { 2id, sugerindo que a frequência das tomas é importante para o efeito. (NE1) }\end{array}$ \\
\hline Wolfe F et al, 1994 & A fluoxetina (20 mg/dia) não demonstrou efeito significativo. (NE 1) \\
\hline Goldenberg D et al, 1996 & $\begin{array}{l}\text { Tanto a fluoxetina ( } 20 \mathrm{mg} / \mathrm{dia}) \text {, como a amitriptilina ( } 25 \mathrm{mg} / \mathrm{dia}) \text { foram associadas a uma melhoria } \\
\text { significativa da dor, do bem-estar geral e da capacidade funcional. O efeito da associação das duas } \\
\text { foi superior ao efeito de cada uma em monoterapia. (NE 1) }\end{array}$ \\
\hline Arnold LM, et al, 2002 & $\begin{array}{l}\text { A fluoxetina (10-80 mg/dia) melhorou a qualidade de vida, a depressão, a dor e a fadiga. O efeito } \\
\text { na dor parece ser independente do efeito no humor. (NE 1) }\end{array}$ \\
\hline Norregaard J et al, 1995 & O citalopram (20-40 mg/dia) não demonstrou efeito. (NE 1) \\
\hline Anderberg UM et la, 2000 & $\begin{array}{l}\text { Verificou-se uma diminuição dos sintomas depressivos com o citalopram (20-40 mg/dia), mas não } \\
\text { houve efeito nos restantes sintomas (NE 1) }\end{array}$ \\
\hline Patkar AA et al, 2007 & A paroxetina (12,5-62,5 mg/dia) melhorou a qualidade de vida, sem redução da dor. (NE 1) \\
\hline Hannonen P et al, 1998 & Moclobemide (450-600 mg/dia) sem efeito significativo. (NE 1) \\
\hline Ginsberg F et al, 1998 & O pirlindol (75 mg, 2id) melhorou significativamente a dor, o $\mathrm{n}^{\circ}$ de pontos dolorosos, o bem-estar \\
\hline
\end{tabular}

Id: número de tomas diárias; n. : número; NE: nível de evidência.

rosos e diversas medidas de qualidade de vida. $\mathrm{O}$ seu efeito na dor pareceu ser independente do seu efeito no humor. ${ }^{4}$

A venlafaxina, outro IRSN, apesar de ter mostrado resultados promissores em dois estudos de pequena dimensão, não mostrou efeito significativo na dor no único EAC incluído na revisão. Este resultado discrepante pode ser devido à baixa dose utilizada de venlafaxina e à curta duração deste último EAC. ${ }^{4}$

O milnacipram, outro IRSN, nas doses de 25-200 $\mathrm{mg} /$ dia, 1 id e 2 id, demonstrou melhorar significativamente a dor. No entanto, o efeito foi superior nos doentes a fazer $2 \mathrm{id}$, sugerindo que a frequência das tomas é importante para o efeito. Este fármaco mostrou também efeito positivo em outras medidas secundárias da fibromialgia. A melhoria dos doentes não deprimidos foi mais significativa do que a dos doentes deprimidos, o que poderá significar que o efeito do milnacipram na dor é independente do seu efeito no humor. No entan- to, este achado precisa ser confirmado num ensaio clínico maior. ${ }^{4}$

A fluoxetina, um ISRS, apesar de não ter demonstrado efeito significativo na dor, na dose $20 \mathrm{mg} 1 \mathrm{id}$, mostrou melhorar a dor, o bem-estar geral e a capacidade funcional, na dose 10-80 mg/dia. ${ }^{4}$

O citalopram, outro ISRS, na dose $20-40 \mathrm{mg} / \mathrm{dia}$, não apresentou diferenças significativas relativamente ao placebo. ${ }^{4}$

Num EAC, a paroxetina, outro ISRS, na dose 12,5$-62,5 \mathrm{mg} / \mathrm{dia}$, reduziu o impacto da fibromialgia na qualidade de vida, apesar de não ter reduzido a intensidade da dor. ${ }^{4}$

O moclobemide, um IMAO, na dose 450-600 mg/dia, não mostrou efeito nos sintomas de fibromialgia. Pelo contrário, o pirlindol, outro IMAO, na dose $75 \mathrm{mg}$ lid, demonstrou melhorar significativamente a dor, o número de pontos dolorosos, o bem-estar geral e o bem-estar físico dos doentes. ${ }^{4}$ 


\section{DISCUSSÃO}

Os antidepressivos tricíclicos foram os primeiros fármacos a ser intensivamente estudados na fibromialgia. Destes, a amitriptilina é aquela com mais estudos realizados. ${ }^{7} \mathrm{O}$ seu benefício é geralmente mais evidente nas duas primeiras semanas de tratamento. Os resultados a longo prazo parecem menos promissores; no entanto, são necessários mais estudos de longa duração para determinar a sua verdadeira eficácia a longo prazo.

A ciclobenzaprina, apesar de partilhar a estrutura tricíclica da amitriptilina, não actua como antidepressivo, mas sim como relaxante muscular. ${ }^{7}$ Este fármaco, que se encontra incluído na classe dos antidepressivos tricíclicos em vários artigos consultados devido à sua semelhança estrutural, demonstrou melhorar a capacidade funcional global do doente e a sua qualidade de sono. A maioria dos estudos, de curta duração, demonstrou um efeito modesto na dor e ausência de efeito na fadiga e nos pontos dolorosos. São, por isso, necessários estudos a longo prazo para determinar a sua verdadeira eficácia.

Tanto a duloxetina, como o milnacipram têm demonstrado resultados promissores. A duloxetina foi aprovada, em Junho de 2008, pela Food and Drug Administration (FDA) para o tratamento da fibromialgia. Esta indicação foi baseada em dois ensaios aleatorizados controlados, duplamente cegos, de fase III. Em ambos os ensaios, ficou demonstrada a eficácia da duloxetina, na dose de $60 \mathrm{mg} /$ dia, no alívio da dor. Foram também observados benefícios em parâmetros secundários, tais como bem-estar geral e impacto na qualidade de vida. A dose recomendada pela FDA é de $60 \mathrm{mg}$, administrada uma vez ao dia. No entanto, o tratamento deve ser iniciado com $30 \mathrm{mg} /$ dia, durante uma semana, aumentando depois para $60 \mathrm{mg} / \mathrm{dia}^{8}$

O milnacipram foi aprovado, pela FDA, para o tratamento da fibromialgia, já em Janeiro de 2009. Esta indicação foi baseada em dois ensaios aleatorizados controlados, duplamente cegos, de fase III, que demonstraram a eficácia do milnacipran, nas doses de 100 $\mathrm{mg} /$ dia e $200 \mathrm{mg} /$ dia, tanto na redução da dor, como na melhoria do bem-estar geral e da capacidade funcional dos doentes com fibromialgia. ${ }^{9}$

Relativamente à venlafaxina, são necessários ensaios clínicos de longa duração, com doses mais elevadas para determinar a sua verdadeira eficácia na fibromialgia.

Não existem ensaios clínicos comparando directamente os SNRI com os antidepressivos tricíclicos, não se sabendo por isso quais os mais eficazes. No entanto, como existem mais estudos sobre os antidepressivos tricíclicos, estes são os mais usados. Os IRSN são uma boa alternativa nos doentes que não tolerem ou não possam tomar antidepressivos tricíclicos.

Os ISRS têm demonstrado resultados inconsistentes. A diversidade de resultados dos diferentes ISRS na fibromialgia sugere que os antidepressivos com efeito selectivo na serotonina são menos eficazes, tanto no alívio da dor, como nos outros sintomas da fibromialgia. O citalopram, que tem uma alta selectividade para os transportadores da serotonina, não se mostrou eficaz na fibromialgia. Pelo contrário, a fluoxetina mostrou ser eficaz e o seu efeito na redução da dor parece ser independente do seu efeito na depressão. A paroxetina, por outro lado, demonstrou ser eficaz na redução do impacto da doença na qualidade de vida, embora se tenha mostrado ineficaz na dor. Os resultados positivos da fluoxetina e da paroxetina sugerem que estes ISRS têm propriedades únicas que lhes proporcionam eficácia nesta doença.

\section{CONCLUSÕES}

A fibromialgia é uma síndrome comum, causadora de grande sofrimento e com grande impacto na qualidade de vida dos doentes.

Existem diversas opções de tratamento para esta síndrome, pelo que é fundamental que o clínico faça a sua escolha com base na melhor evidência científica.

Assim, segundo a evidência actualmente disponível, os antidepressivos com benefício no tratamento da fibromialgia são a amitriptilina (SORT A), a duloxetina (SORT A), o milnacipram (SORT A), a fluoxetina (SORT B) e o pirlindol (SORT B). A ciclobenzaprina, apesar de não actuar como antidepressivo, partilha da estrutura tricíclica da amitriptilina e demonstrou ser benéfico no tratamento da fibromialgia (SORT A).

São necessários ensaios aleatorizados controlados comparando as diferentes classes de antidepressivos entre si, de forma a determinar a sua eficácia relativa. São também necessários ensaios aleatorizados controlados de longa duração, de forma a determinar a eficá- 
cia a longo prazo dos diferentes fármacos nos diversos sintomas existentes na fibromialgia.

\section{REFERÊNCIAS BIBLIOGRÁFICAS}

1. Carville SF, Arendt-Nielsen S, Bliddal H, Blotman F, Branco JC, Buskila $D$, et al. Eular evidence-based recommendations for the management of fibromyalgia syndrome. Ann Rheum Dis 2008 Apr; 67 (4): 536-41.

2. Goldenberg LD, Burckhardt C, Crofford L. Management of fibromyalgia syndrome. JAMA 2004 Nov 17; 292 (19): 2388-95.

3. O Malley PG, Balden E, Tomkins G, Santoro J, Kroenke K, Jackson JL. Treatment of fibromyalgia with antidepressants: a meta-analysis. J Gen Intern Med 2000 Sep; 15 (9): 659-66.

4. Arnold LM, Keck PE Jr, Welge JA. Antidepressant treatment of fibromyalgia: a meta-analysis and review. Psychosomatics 2000 Mar-Apr; 41 (2):104-13.

5. Tofferi JK, Jackson JL, O Malley PG. Treatment of fibromyalgia with ciclobenzaprine: a meta-analysis. Arthritis Rheum 2004 Feb; 51 (1): 9-13.

6. Arnold LM. Duloxetine and other antidepressants in the treatment of patients with fibromyalgia. Pain Med 2007 Sep; 8 Suppl 2: S63-74.
7. Abeles M, Solitar BM, Pillinger MH, Abeles AM. Update on fibromyalgia therapy. Am J Med 2008 Jul; 121 (7): 555-61.

8. Cymbalta approved for fibromyalgia. Medscape Medical News. 2008. Disponível em: http://cme.medscape.com/viewarticle/576320 [acedido em 30/12/2009].

9. FDA Approves milnacipran for fibromyalgia. Medscape Medical News, Alerts, Approvals and Safety Changes. 2009. Disponível em: http://cme.medscape.com/viewarticle/587170 [acedido em 30/12/2009].

Os autores declararam não possuir conflitos de interesses

\author{
ENDEREÇO PARA CORRESPONDÊNCIA \\ Sofia Almeida \\ Avenida Gago Coutinho, n. 1531 \\ 4405-710 Gulpilhares, Vila Nova de Gaia \\ E-mail: anasofiaalmeida@netcabo.pt
}

Recebido em 29/05/2009

Aceite para publicação em 21/11/2009

\section{ABSTRACT}

\section{THERAPEUTIC ROLE OF ANTIDEPRESSANTS IN FIBROMYALGIA}

Introduction: Fibromyalgia is characterized by chronic widespread pain of unknown etiology associated with fatigue, stiffness, sleep disturbance, depression, anxiety, headache, migraine, variable bowel habits, abdominal pain and urinary frequency. The 1990 American College of Rheumatology criteria for fibromyalgia include widespread pain and the presence of excessive tenderness on applying pressure to 11 of 18 specific muscle-tendon sites. Its prevalence is $2 \%$ in the United States.

Objective: Review of recent evidence on the role of antidepressants in fibromyalgia.

Data sources: Medline/PubMed and evidence-based medicine sites.

Review methods: Research of meta-analyses (MA) and systematic reviews (SR) published between 2000 and July 2008, in Portuguese and English, using the key-words Fibromyalgia, Antidepressants, Fibromyalgia Treatment. The SORT taxonomy was used to present the results.

Results: From the 10 articles found, 3 MA and 4 SR were selected. Analysis showed that the effect of tricyclic antidepressants in fibromyalgia is moderate, being higher in sleep and reduced in pain and stiffness. The cyclobenzaprine, which has a similar tricyclic structure to amitriptyline, although it performs as a muscle relaxant and not as an antidepressant, improves global function of patients, sleep, and less significantly, the pain. Regarding serotonin and norepinehrine reuptake inhibitors, the milnacipram and duloxetine are effective in pain and qulity of life, while venlafaxine seem to be effective in higher doses. Of the serotonin reuptake inhibitors, fluoxetine is effective in pain, well being and depression, paroxetine reduces the impact of the disease on the quality of life, with no effect on pain and citalopram is ineffective. Regarding monoamine oxidase inhibitors, the pirlindol improves pain and well being of patients, while moclobemide is ineffective.

Conclusions: The antidepressants with proven benefit in treating fibromyalgia are amitriptyline, duloxetine, milnacipram (SORT A), fluoxetine and pirlindol (SORT B). Also cyclobenzaprine proved efficacy in treating fibromyalgia (SORT A). Long duration studies are needed to determine their benefit in the long term.

Keywords: Fibromyalgia; Antidepressants; Fibromyalgia Treatment. 